\title{
ABELIAN QUOTIENTS OF THE MAPPING CLASS GROUP OF A 2-MANIFOLD
}

\author{
BY JOAN S. BIRMAN
}

Communicated by Raoul Bott, July 7, 1969

Let $T_{g}$ be a closed, orientable 2-manifold of genus $g$, and let $M_{\boldsymbol{g}}$ be the mapping class group of $T_{0}$, that is the group of orientationpreserving homeomorphisms of $T_{0} \rightarrow T_{0}$ modulo those isotopic to the identity. The following theorem was proved by D. Mumford in [6]: If $\left[M_{\theta}, M_{\theta}\right]$ is the commutator subgroup of $M_{g}$, then $A_{g}=M_{\theta} /\left[M_{g}, M_{\theta}\right]$ is a finite cyclic group whose order is a divisor of 10 . We give a very brief and elementary reproof of Mumford's theorem, and at the same time improve his result to show that the order of $A_{g}$ is 2 if $g \geqq 3$.

Generators for $M_{0}$ are well known, and a particularly convenient set is given by W. B. R. Lickorish in [3]. Lickorish's generators are "screw maps" about closed curves on the surface $T_{0}$ (the definition of a screw map is the same as that in [6]), and Lickorish shows that the screw maps about the curves $\left\{u_{i}, z_{i}, c_{i} ; 1 \leqq i \leqq g, 1 \leqq j \leqq g-1\right\}$ in Figure 1 generate $M_{0}$.

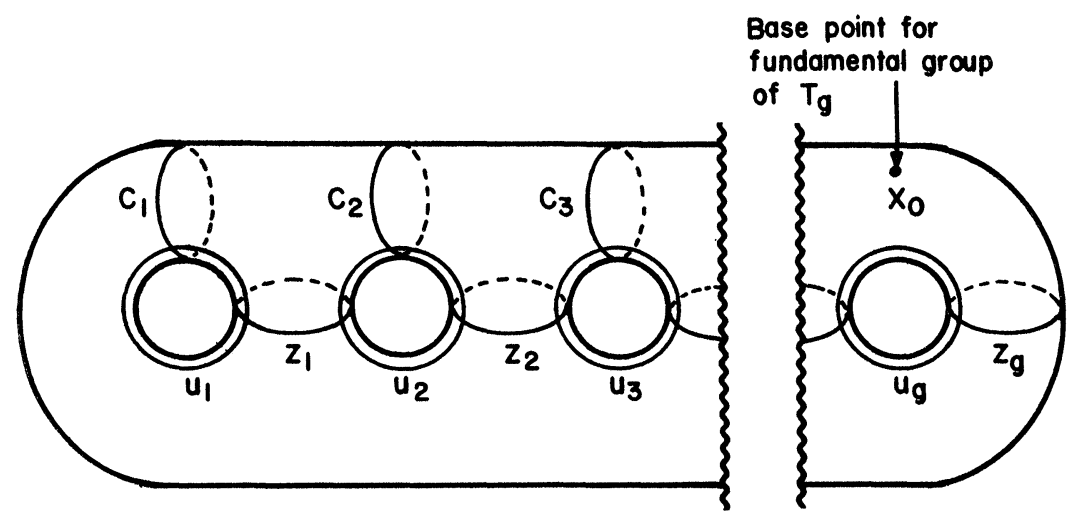

FIGURE 1

By a well-known result [5] the group $M_{\theta}$ is isomorphic to a group of automorphism classes (cosets of the subgroup of inner automorphisms in the group of all automorphisms) of the fundamental group

AMS Subject Classifications. Primary 3045, 2022; Secondary 3080, 1451.

Key Words and Phrases. Mapping class group, homeotopy groups, automorphism group of the fundamental group of a 2-manifold, Teichmuller modular group, screw map, twist map. 
$\pi_{1} T_{0}$ of the surface $T_{0}$. Choosing generators $\left\{t_{i}, s_{i} ; 1 \leqq i \leqq g\right\}$ for $\pi_{1} T_{0}$ as illustrated in Figure 2, and denoting screw maps about the curves $u_{i}, z_{i}$ and $c_{i}$ by $U_{i}, Z_{i}$ and $C_{i}$ respectively, the automorphisms of $\pi_{1} T_{\theta}$ corresponding to Lickorish's generators of $M_{0}$ are easily determined (see [1]), and are given explicitly as follows:

$$
\begin{aligned}
& U_{i}: t_{i} \rightarrow t_{i} s_{i} \quad 1 \leqq i \leqq g \\
& Z_{i}: s_{i} \rightarrow t_{i}^{-1} t_{i+1} s_{i} \quad 1 \leqq i \leqq g-1 \\
& s_{i+1} \rightarrow s_{i+1} t_{i+1}^{-1} t_{i} \quad 1 \leqq i \leqq g-1 \\
& Z_{g}: s_{\theta} \rightarrow t_{g}^{-1} s_{g} \\
& C_{i}: s_{j} \rightarrow t_{i} s_{j} t_{i}^{-1} \\
& j<i \\
& t_{j} \rightarrow t_{i} t_{j} t_{i}^{-1} \\
& j<i \\
& 1 \leqq i \leqq g-1 \\
& s_{i} \rightarrow s_{i} t_{i}^{-1}
\end{aligned}
$$

where it is understood that every generator of $\pi_{1} T_{0}$ which is not listed explicitly is unaltered by the screw maps.

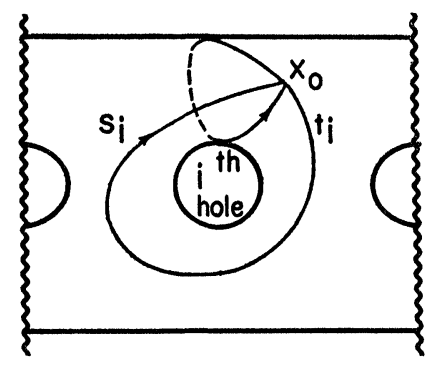

FIGURE 2

This representation of $M_{\theta}$ as a group of automorphism classes provides a very simple tool for calculation in $M_{g}$. If one suspects that two sequences of screw maps are equivalent in $M_{\theta}$, one simply calculates the induced automorphisms, and determines if they agree modulo an inner automorphism. Using this procedure, the following relations can be verified to hold in $M_{g}$ :
(4) $\quad U_{i} Z_{i} U_{i}=Z_{i} U_{i} Z_{i}$
$1 \leqq i \leqq g$
(5) $\quad U_{i+1} Z_{i} U_{i+1}=Z_{i} U_{i+1} Z_{i}$
$1 \leqq i \leqq g-1$ 
(6) $\quad C_{i} U_{i} C_{i}=U_{i} C_{i} U_{i} \quad 1 \leqq i \leqq g$

(7) $\quad\left(C_{1} U_{1} Z_{1} U_{2} Z_{2} \cdots U_{0} Z_{0}^{2} U_{0} \cdots Z_{2} U_{2} Z_{1} U_{1} C_{1}\right)^{2}=1$

(8) $\quad\left(C_{1} U_{1} Z_{1} U_{2} Z_{2} \cdots U_{0} Z_{0}\right)^{20+1}=1$

(9) $\left(U_{1} Z_{1} U_{2} C_{2}\right)^{5}=\left(C_{1} U_{1} Z_{1} U_{2} C_{2}^{2} U_{2} Z_{1} U_{1} C_{1}\right) \quad$ if $g \geqq 3$

(10) $\left(U_{1} Z_{1} U_{2} Z_{2} U_{3} C_{3}\right)^{7}=\left(C_{1} U_{1} Z_{1} U_{2} Z_{2} U_{3} C_{3}^{2} U_{3} Z_{2} U_{2} Z_{1} U_{1} C_{1}\right)$ if $g \geqq 4$.

Relations (4)-(8) above were determined by the author in [1]; relations (9) and (10) are new, to the author's knowledge.

We now consider the abelianizing homomorphism $\alpha: M_{0} \rightarrow A_{0}$. Under $\alpha$, relation (4) goes over to

$$
\alpha\left(U_{i}\right) \alpha\left(Z_{i}\right) \alpha\left(U_{i}\right)=\alpha\left(Z_{i}\right) \alpha\left(U_{i}\right) \alpha\left(Z_{i}\right) .
$$

Since all elements in $A_{\theta}$ commute, (11) implies:

$$
\alpha\left(U_{i}\right)=\alpha\left(Z_{i}\right) .
$$

Since similar relations link the entire set of generators of $M_{\theta}$, we obtain immediately that $A_{\boldsymbol{\sigma}}$ is a cyclic group. Denoting the single generator of $A_{o}$ by $h=\alpha\left(U_{i}\right)$, equations (7), (8), (9) and (10) then give

$$
\begin{aligned}
h^{(20+1)(4)} & =h^{(20+1)(20+2)}=1 \quad \text { for all } g, \\
h^{10} & =1 \quad \text { if } g \geqq 3, \\
h^{28} & =1 \quad \text { if } g \geqq 4 .
\end{aligned}
$$

Together these imply that the order of $h$ is a divisor of 10 if $g=2$, while for $g \geqq 3$ the order of $h$ divides 2 .

It only remains to prove that the order of $A_{g}$ cannot be 1 . To establish this, we make use of the well-known fact that the group $\mathrm{Sp}(2 g, Z)$ of $2 g$-by- $2 g$ symplectic matrices with integral entries is a quotient group of $M_{0}$ [5], and hence the commutator quotient group of $\operatorname{Sp}(2 g, Z)$ is a quotient group of $A_{g}$. The author is grateful to J. Mennicke for pointing out that it follows from known work [2] that the commutator quotient group of $\operatorname{Sp}(2 g, Z)$ is of order 2; hence $A_{g}$ is of order 2 for all $g \geqq 3$. For $g=2$ it is known that $A_{g}$ is cyclic of order 10.

Some geometric insight into the proof outlined above is obtained by noting that the cyclic nature of $A_{g}$ is an immediate consequence of relations (4), (5), (6). For the case of the torus $(g=1)$ these reduce to the single relation:

$$
U_{1} Z_{1} U_{1}=Z_{1} U_{1} Z_{1}
$$

which is classical. Now, it is easily established that this relation re- 
mains valid on a torus with $n$ points removed. Since all pairs $\left(U_{1}, Z_{i}\right)$, $\left(U_{i}, C_{i}\right)$ and $\left(Z_{i}, U_{i+1}\right)$ of generators of $M_{g}$ can be displayed as appropriate pairs of screw maps on subsets of $T_{g}$ which are homeomorphic to a punctured torus, relations (4), (5), and (6) are seen to follow directly from the corresponding relation in $M_{1}$. The order of the single generator of $A_{o}$ is determined by relations (7), (8), (9), and (10). Of these, relations (7) and (8) basically express symmetries in the geometric realization of the surface $T_{g}$; relations (9) and (10) are obtained from (7) and (8) specialized to the cases $g=2$ and 3 respectively, and carried over to subsets of $T_{g}$ which are homeomorphic to ( $T_{2}$-one point) and $\left(T_{3}\right.$-one point) respectively.

\section{REFERENCES}

1. Joan Birman, Automorphisms of the fundamental group of a closed, orientable 2-manifold, Proc. Amer. Math. Soc. 21 (1969), 351-354.

2. Helmut Klingen, Charakterisierung der Siegelschen Modulgruppe durch ein endliches System definierender Relationen, Math. Ann. 144 (1961), 64-82. MR 24 \#A3137.

3. W. B. R. Lickorish, $A$ finite set of generators for the homeotopy group of a 2-manifold, Proc. Cambridge Philos. Soc. 60 (1964), 769-778. MR 30 \#1500.

4. W. Magnus, Über Automorphismen von Fundamentalgruppen berandeter Flächen, Math. Ann. 109 (1934), 617.

5. W. Magnus, A. Karrass and D. Solitar, Combinatorial group theory. Presentations of groups in terms of generators and relations, Pure and Appl. Math., vol. 13, Interscience, New York, 1966, pp. 175-178. MR 34 \#7617.

6. D. Mumford, Abelian quotients of the Teichmiiller modular group, J. Analyse Math. 18 (1967), 227-244. MR $36 \# 2623$.

Stevens Institute of Technology, Hoboken, New Jersey 07030 